\title{
Laser-excited ultrahigh-resolution photoemission spectroscopy of superconducting $\mathrm{Na}_{0.35} \mathrm{CoO}_{2} \cdot 1.3 \mathrm{H}_{2} \mathrm{O}$
}

T. Shimojima, ${ }^{1}$ T. Yokoya, ${ }^{1, *}$ T. Kiss, ${ }^{1}$ A. Chainani, ${ }^{2}$ S. Shin, ${ }^{1,2}$ T. Togashi, ${ }^{2}$ C. Chen, ${ }^{3}$

S. Watanabe, ${ }^{1 ⿴}$ K. Takada, ${ }^{4}$ T. Sasaki, ${ }^{4}$ H. Sakurai, ${ }^{5}$ and E. Takayama-Muromachi ${ }^{5}$

${ }^{1}$ Institute for Solid State Physics (ISSP), University of Tokyo, Kashiwa, Chiba 277-8681, Japan

${ }^{2}$ The Institute of Physical and Chemical Research (RIKEN),Sayo-gun, Hyogo 679-5143, Japan

${ }^{3}$ Beijing Center for Crystal R\&D, Chinese Academy of Science Zhongguancun,Beijing 100080, China

${ }^{4}$ Advanced Materials Laboratory, National Institute for Materials Science, Tsukuba, Ibaraki 305-0044, Japan.

${ }^{5}$ Superconducting Materials Center, National Institute for Materials Science, Tsukuba, Ibaraki 305-0044, Japan.

"Present address: Japan Synchrotron Radiation Research Institute (JASRI), Spring8, Sayo, Hyogo 679-5198, Japan

Abstract

We have studied the electronic structure near the Fermi level of the layered cobaltate superconductor $\mathrm{Na}_{0.35} \mathrm{CoO}_{2} \cdot 1.3 \mathrm{H}_{2} \mathrm{O}$ using laser-excited ultrahigh-resolution photoemission spectroscopy (PES). In the normal state, we observed the formation of a pseudogap with an energy scale of $\sim 20 \mathrm{meV}$. Across the superconducting transition, we observed the leading edge shift at $3.5 \mathrm{~K}$ compared to that at $10 \mathrm{~K}$, indicating surperconducting gap (SC gap) 
opening having a gap value of $0.5-0.7 \mathrm{meV}$ depending models used. The energy scale of the SC gap is $\sim 20$ times smaller than that of the pseudogap, suggesting existence of competing order parameters at low temperatures.

\section{Introduction}

Recently, Takada et al. has reported the first cobalt oxide superconductor $\mathrm{Na}_{0.35} \mathrm{CoO}_{2} \cdot 1.3 \mathrm{H}_{2} \mathrm{O}$ (the transition temperature $T_{c}$ of $\sim 4.5 \mathrm{~K}$ ). ${ }^{1}$ It has two-dimensional conductive $\mathrm{CoO}_{2}$ planes which can be regarded as an electron-doped correlated $S=1 / 2$ triangular network of frustrated Co spins, ${ }^{1}$ where novel superconductivity emerging from a non Fermi-liquid ground state has been proposed. ${ }^{2}$ In the cuprate high- $T_{c}$ 's, the $\mathrm{CuO}_{2}$ plain is described in terms of a quasi-two dimensional correlated system. The characteristics of $\mathrm{Na}_{0.35} \mathrm{CoO}_{2} \cdot 1.3 \mathrm{H}_{2} \mathrm{O}$ resemble those of high- $T_{c}$ 's, except for the lattice structure of conductive layers: triangular or square. Comparison between the two superconductors will be meaningful for deeper understanding of the unconventional superconductivity in layered transition metal oxides.

After the discovery, extensive experimental and theoretical studies have been made in order to understand the origin of the superconductivity of $\mathrm{Na}_{0.35} \mathrm{CoO}_{2} \cdot 1.3 \mathrm{H}_{2} \mathrm{O}$. Theoretically, several order parameters have been proposed depending on the model used ${ }^{3}$. For instance, a d-wave order parameter derived from spin fluctuations using a t-J model on the triangular lattice ${ }^{4}$ and a 
f-wave one derived from charge fluctuations using a single-band extended Hubbard model ${ }^{5}$ have been proposed. The former indicates that $d_{1}+i_{2}$ superconductivity is the neighboring phase of spin gap phase. The latter indicates that f-wave superconductivity is realized in the vicinity of charge density wave (CDW) instability in the triangular lattice. In addition, experimental results have also contradicted with each other. Kobayashi et al. reported a coherence peak just below $T_{c}$ in the spin-lattice relaxation rate $\left(1 / \mathrm{T}_{1}\right)$ curve by nuclear quadrupole resonance (NQR), indicating the fully gapped superconductor ${ }^{7}$. On the other hand, by NQR, Fujimoto et al. and Ishida et al. reported no coherence peak, showing existence of line-nodes in the gap function ${ }^{8,9}$. Thus paring symmetry is still under controversy.

On the other hand, a direct observation of a SC gap by PES can provide significant insight into the paring symmetry. Recently, we have constructed a newly designed photoemission spectrometer with a CW ultraviolet laser $(\mathrm{h}=6.994 \mathrm{eV})$ as an excitation source. For investigating electronic structure of samples having a very delicate surface and a very small energy scale, PES with a lower photon energy provided by a laser was found to be very powerful, ${ }^{10}$ because of the very high energy resolution and the expected large escape depth ${ }^{11}$. In this paper, we report the temperature-dependent electronic structure near $E_{\mathrm{F}}$ of polycrystalline $\mathrm{Na}_{0.35} \mathrm{CoO}_{2} \cdot 1.3 \mathrm{H}_{2} \mathrm{O}$ studied from PES using an ultraviolet laser. We observed SC gap opening across $\mathrm{T}_{\mathrm{c}}$ with an unusual reduction of the intensity at $E_{\mathrm{F}}$ in the normal state. 
We also performed numerical simulations using Dynes function ${ }^{12}$ for the spectrum at $3.5 \mathrm{~K}$ in order to determine the magnitude and pairing symmetry of the SC gap,

\section{Experimental}

Polycrystalline samples of $\mathrm{Na}_{0.35} \mathrm{CoO}_{2} \cdot 1.3 \mathrm{H}_{2} \mathrm{O}$ is synthesized from $\mathrm{Na}_{0.7} \mathrm{CoO}_{2}$ through a chemical oxidation process, by which a part of $\mathrm{Na}$ ions is removed and water molecules are intercalated between $\mathrm{CoO}_{2}$ and $\mathrm{Na}$ planes. ${ }^{1}$ The partial reduction of water easily produces non-superconducting $\mathrm{Na}_{0.35} \mathrm{CoO}_{2} \cdot 0.7 \mathrm{H}_{2} \mathrm{O}$. Hydrated samples are very sensitive to humidity. PES measurements under an ultrahigh vacuum condition require carefully handling of them. We handled hydrated samples as follows. First, the samples are covered with silver paste and mounted them on copper substrates to prevent loss of water molecules under vacuum. Then, the prepared samples were cooled to $\sim 180 \mathrm{~K}$ and are fractured in-situ. After the fracturing, they are transferred to a measurement chamber and measured without warming up above $\sim 180$ $\mathrm{K}$. We chose the temperature of $180 \mathrm{~K}$ because no loss of water molecules occurs in $\mathrm{Na}_{0.35} \mathrm{CoO}_{2} \cdot 1.3 \mathrm{H}_{2} \mathrm{O}$ below $250 \mathrm{~K} .{ }^{13}$

All PES measurements were performed on a spectrometer built using a GAMMADATA-SCIENTA R-4000 electron analyzer and an ultraviolet laser. The best total energy resolution is $360 \mathrm{eV} \cdot{ }^{10}$ However, the energy resolution for SC gap and near- $E_{\mathrm{F}}$ 
measurements were set to $1.8 \mathrm{meV}$ and $6.5 \mathrm{meV}$ respectively, in order to get reasonable count rate. Samples are cooled using a flowing liquid He refrigerator with improved thermal shieldings. The base pressure of the measurement chamber was better than $2 \times 10^{-11}$ Torr. The spectra were all reproducible during the measurement.

\section{Results and discussion}

Shown in Fig. 1. are (a) the PES spectra near $E_{\mathrm{F}}$ of $\mathrm{Na}_{0.35} \mathrm{CoO}_{2} \cdot 1.3 \mathrm{H}_{2} \mathrm{O}$ measured as a function of temperature from $3.5 \mathrm{~K}$ to $150 \mathrm{~K}$, (b) the enlargement of the near $E_{\mathrm{F}}$ region, which highlights the difference in temperature dependent change at $E_{\mathrm{F}}$, and (c) the normalized density of state (DOS) of $\mathrm{Na}_{0.35} \mathrm{CoO}_{2} \cdot 1.3 \mathrm{H}_{2} \mathrm{O}$. In Fig. 1. (a), the spectra show systematic temperature dependence with a clear Fermi edge structure at lower temperatures. However, we found that there is an additional decrease within $20 \mathrm{meV}$ binding energy of $E_{\mathrm{F}}$ in the spectrum at $3.5 \mathrm{~K}$ (black arrow). In addition, small but important reduction of intensity at $E_{\mathrm{F}}$ is also observed in the raw spectra (Fig. 1.(b)). Thus, the raw data shows an unusual normal-state electronic structure change near $E_{\mathrm{F}}$. In order to see the clear temperature-dependent change in DOS, we extracted the effect of the Fermi-Dirac (FD) function from the raw data as follows. The raw spectrum at each temperature was divided by the corresponding the FD function convoluted with a Gaussian with the instrumental resolution. The divided spectra at all 
temperatures were further divided with the smoothed spectrum at $150 \mathrm{~K} \cdot{ }^{14}$ As shown in Fig. 1 (c), the normalized DOS of $\mathrm{Na}_{0.35} \mathrm{CoO}_{2} \cdot 1.3 \mathrm{H}_{2} \mathrm{O}$ within $20 \mathrm{meV}$ binding energy of $E_{\mathrm{F}}$ are gradually suppressed with decreasing temperature, indicative of formation of a pseudogap with an energy scale of $20 \mathrm{meV}$ is formed. Further, the reduced spectral weight around $E_{\mathrm{F}}$ seems to be compensated by a slight increase beyond $20 \mathrm{meV}$ binding energy. It indicates that spectral weight is transferred from the near- $E_{\mathrm{F}}$ region to the higher-binding energy region. Recently temperature-dependent pseudogap in non-hydrated samples $\mathrm{Na}_{\mathrm{x}} \mathrm{CoO}_{2}(\mathrm{x}=0.5,0.25)$ was reported from optical spectroscopy ${ }^{15}$. The energy scales of half of the pseudogap measured by optical spectroscopy are 6-25 meV depending on the $\mathrm{Na}$ concentration. These values are consistent with the pseudogap size of present study. However the reduction of normalized DOS in hydrated samples is smaller $(10-20 \%$ of the spectrum at $150 \mathrm{~K})$ than the optical data. It may indicate that the pseudogap formation is partially suppressed due to the difference in $\mathrm{Na}$ concentration and/or inclusion of the water molecules.

Figure 2 shows the ultrahigh resolution PES spectra measured at $3.5 \mathrm{~K}$ (superconducting phase, black circle) and $10 \mathrm{~K}$ (normal phase, gray circle). The broken lines denote the FD functions at $3.5 \mathrm{~K}$ and $10 \mathrm{~K}$, convoluted with the Gaussian corresponding to the instrumental resolution. We found that, while the spectrum at $10 \mathrm{~K}$ is well reproduced by the FD function at $10 \mathrm{~K}$, spectrum of $3.5 \mathrm{~K}$ shows a slight leading-edge shift and seems not to be fitted by the FD 
function. Spectral difference between the spectrum at $3.5 \mathrm{~K}$ and $10 \mathrm{~K}$ is also shown as gray area on a lower part of Fig. 2. Assuming that the both spectra are well reproduced by the FD function at each temperature, the difference should be zero at $E_{\mathrm{F}}$. However, the intensity at $E_{\mathrm{F}}$ of the different spectrum is not zero, and the crossing point between the difference spectra and the horizontal line indicating intensity zero shifts to higher binding energy. This indicates that there is SC gap opening. Absence of a sharp peak is due to the relatively smaller SC gap compared with the energy resolution used as confirmed below.

In order to obtain the magnitude of the SC gap and determine the pairing symmetry, we have done numerical simulations using Dynes functions as shown in Fig. 3. Figure 3 shows the ultrahigh resolution spectra of $\mathrm{Na}_{0.35} \mathrm{CoO}_{2} \cdot 1.3 \mathrm{H}_{2} \mathrm{O}$ measured at $3.5 \mathrm{~K}$ (open circle), the FD function at 3.5K (broken line), and fitting curves with Dynes function (black and gray lines). From Fig. 3, it seems that the FD function failed to reproduce the spectrum at $3.5 \mathrm{~K}$. We used Dynes functions with s-wave and d-wave symmetry to fit the spectra. Better fittings have been obtained with $=0.5 \pm 0.1 \mathrm{meV}$ and $=0.05 \mathrm{meV}$ for s-wave (black line), $=0.7 \pm 0.1 \mathrm{meV}$ and $=0.05 \mathrm{meV}$ for d-wave (gray line), where is SC gap size, a term of quasi-particle lifetime. It is difficult to determine the pairing symmetry from the fitting curves, since they give little difference due to the insufficient energy resolution. However, both fitting curves reproduce the raw spectrum better than the FD function. The obtained values of $(3.5 \mathrm{~K})=0.5$ 
meV (s-wave) and $(3.5 \mathrm{~K})=0.7 \mathrm{meV}\left(\mathrm{d}\right.$-wave) correspond to $2(0) / \mathrm{k}_{\mathrm{B}} \mathrm{T}_{\mathrm{c}} \sim 3.4$ and $2(0) / \mathrm{k}_{\mathrm{B}} \mathrm{T}_{\mathrm{c}} \sim$ 4.8, respectively (the known mean field relation of $2 / \mathrm{k}_{\mathrm{B}} \mathrm{T}_{\mathrm{c}}=3.54$ according to BCS theory, where $k_{B}$ is the Boltzmann constant). From NQR measurements, the $1 / \mathrm{T}_{1}$ curve was reproduced by the? ?calculation using the two-dimensional line-node model $(\theta)=(0) \cos (2 \theta)$. The appropriate fitting parameters were reported to be $2(0) / \mathrm{k}_{\mathrm{B}} \mathrm{T}_{\mathrm{c}}=3.5$ by Ishida et al. ${ }^{9}$ and $2(0) / \mathrm{k}_{\mathrm{B}} \mathrm{T}_{\mathrm{c}}=5.2$ by Fujimoto et al. ${ }^{8}$, respectively. The present PES value from the d-wave model is located between these two values. This is the first observation of SC gap of $\mathrm{Na}_{0.35} \mathrm{CoO}_{2} \cdot 1.3 \mathrm{H}_{2} \mathrm{O}$ measured by ultrahigh resolution PES. In the present study, we could not use the highest resolution due to the very low count rate near $E_{\mathrm{F}}$, which is inherent to the cobalt oxides. We are now preparing laser PES studies of single crystal samples, and this will give more detailed information on the pairing symmetry.

Lastly we discuss the relation between the superconducting gap and pseudogap. The energy scale of the obtained SC gap $(0.5-0.7 \mathrm{meV})$ is smaller than the pseudogap $(20 \mathrm{meV})$ of $\mathrm{Na}_{0.35} \mathrm{CoO}_{2} \cdot 1.3 \mathrm{H}_{2} \mathrm{O}$. This indicates that the pseudogap is the different phenomena from superconductivity. Such a large pseudogap is also reported on high- $T_{c}$ 's ${ }^{16,17}$, where it is found that pseudogap formation is related to the growth of magnetic correlations. On the other hand, a temperature-dependent pseudogap has been observed in $\mathrm{Ba}_{0.67} \mathrm{~K}_{0.33} \mathrm{BiO}_{3},{ }^{18}$ where the energy 
scale of the pseudogap correlated with the energy of the breathing mode phonon. In the Co oxides, the energy scale of $20 \mathrm{meV}$ is comparable to the nearest neighboring magnetic coupling constant $J=10-20 \mathrm{meV}^{19}$ which has been also concluded from angle-resolved PES measurements. ${ }^{20,21}$ This $J$ value is smaller than the highest phonon energies. ${ }^{15}$ Thus, while additional studies are needed to clarify the relation between the superconductivity and the formation of the pseudogap, there is a possibility of existence of another ground state competing with and/or cooperating the superconductivity at low temperature in the new layered Co oxide superconductor.

In conclusion, we performed ultra-igh resolution PES and observed pseudogap formation with the energy scale of $\sim 20 \mathrm{meV}$ in normalized DOS of $\mathrm{Na}_{0.35} \mathrm{CoO}_{2} \cdot 1 \cdot 3 \mathrm{H}_{2} \mathrm{O}$. We also observed the leading edge shift of $\mathrm{Na}_{0.35} \mathrm{CoO}_{2} \cdot 1.3 \mathrm{H}_{2} \mathrm{O}$ at $3.5 \mathrm{~K}$, indicating SC gap opening. Numerical simulation with Dynes function gives the SC gap size of 0.5 (s-wave) and $0.7 \mathrm{meV}$ (d-wave), corresponding to $2(0) / \mathrm{k}_{\mathrm{B}} \mathrm{T}_{\mathrm{c}} \sim 3.4$ and 4.8 , respectively.

We thank S. Maekawa, W. Koshibae and Y. Yanase for their valuable discussion. We also thank F. Kanetaka for his help in PES measurements. This study was partially supported by CREST of Japan Science and Technology Agency (JST), and by Grants-in-Aid for Scientific 
Research (B) (16340111) and Young Scientists (A) (14702010) from Japan Society for the Promotion of Science.

\section{References}

${ }^{1}$ K. Takada et al., Nature, 422, 53 (2003).

${ }^{2}$ P. W. Anderson, Science 235, 1196 (1987).

${ }^{3}$ G. Baskaran, Phys. Rev. Lett. 91, 097003 (2003); A. Tanaka et al., Phys. Rev. Lett. 91, 
257006 (2003); K. Kuroki et al., cond-mat/0311619; M. Ogata J. Phys. Soc. Japan 72, 1839 (2003); B. Kumar et al., Phys. Rev. B 68, 104508 (2003); D. J. Singh et al., Phys. Rev. B 68, 020503 (2003); C. Honerkamp et al., Phys. Rev. B 68, 104510 (2003); Q. H. Wang et al., cond-mat/0304377; Y. Tanaka et al., cond-mat/0311266.

${ }^{4}$ G. Baskaran, Phys. Rev. Lett. 91, 097003 (2003)

${ }^{5}$ Y. Tanaka et al., cond-mat/0311266.

${ }^{6}$ Y. Kobayashi et al., J. Phys. Soc. Jpn 722161 (2003); T. Waki et al., cond-mat/0306036; Y. Kobayashi et al., J. Phys. Soc. Jpn 722453 (2003); T. Fujimoto et al., Phys. Rev. Lett. 92, 047004 (2004); K. Ishida et al., J. Phys. Soc. Jpn 72, 3041 (2003); W. Higemoto et al., cond-mat/0310324; A. Kanigel et al., cond-mat/0311427.

${ }^{7}$ Y. Kobayashi et al., J. Phys. Soc. Jpn 722161 (2003)

${ }^{8}$ T. Fujimoto et al., Phys. Rev. Lett. 92, 047004 (2004)

${ }^{9}$ K. Ishida et al., J. Phys. Soc. Jpn 72, 3041 (2003)

${ }^{10}$ T. Kiss et al., Submitted.

${ }^{11}$ M. P. Seah and W. A. Dench, Surface Interface Anal. 1, 2 (1979).

${ }^{12}$ R. C. Dynes et al., PRL 41, 1509 (1978).

${ }^{13}$ H. Sakurai, private communications.

${ }^{14}$ This series of processes gives a good approximation for the structure in the DOS larger than the energy resolution $(6.5 \mathrm{meV})$, putting the DOS at $150 \mathrm{~K}$ as a standard. 
${ }^{15}$ J. Hwang et al., cond-mat/0405200.

${ }^{16}$ T. Takahashi et al., J. Phys. Chem. Solids 62, 41 (2001).

${ }^{17}$ T. Sato et al., Phys. Rev. Lett. 83, 2254 (1999).

${ }^{18}$ A. Chainani et al., Phys. Rev. B 64, 180509 (2001).

${ }^{19}$ J. Kunes, K.-W. Lee, and W. E. Pickett, cond-mat/0308388; K.-W. Lee, J. Kunes, and W. E. Pickett, Phys. Rev. B 70, 045104 (2004).

${ }^{20}$ M. Z. Hasan et al., Phys. Rev. Lett. 92, 246402 (2004).

${ }^{21}$ H.-B. Yang et al., Phys. Rev. Lett. 92, 246403 (2004).

\section{Figure Legends}

Fig. 1. (a) Temperature-dependent spectra of $\mathrm{Na}_{0.35} \mathrm{CoO}_{2} \cdot 1.3 \mathrm{H}_{2} \mathrm{O}$ measured with $6.994 \mathrm{eV}$ photon energy. (b) The enlargement of the near $E_{\mathrm{F}}$ region, which highlights the difference in temperature dependent change at $E_{\mathrm{F}}$. (c) The normalized DOS of $\mathrm{Na}_{0.35} \mathrm{CoO}_{2} \cdot 1.3 \mathrm{H}_{2} \mathrm{O}$, showing 
pseudogap formation at lower temperature.

Fig. 2. Ultrahigh resolution spectrum of $\mathrm{Na}_{0.35} \mathrm{CoO}_{2} \cdot 1.3 \mathrm{H}_{2} \mathrm{O}$ measured at $3.5 \mathrm{~K}$ (superconducting phase, black circles) and $10 \mathrm{~K}$ (normal phase, gray circles). The broken lines denote the FD functions at $3.5 \mathrm{~K}$ and $10 \mathrm{~K}$, convoluted with the Gaussian with the instrumental resolution of $1.8 \mathrm{meV}$. Gray area is shown as a spectral difference between spectrum at $3.5 \mathrm{~K}$ and $10 \mathrm{~K}$.

Fig.3. Ultrahigh resolution spectrum of $\mathrm{Na}_{0.35} \mathrm{CoO}_{2} \cdot 1.3 \mathrm{H}_{2} \mathrm{O}$ measured at $3.5 \mathrm{~K}$ (open circles) compared with fitting results. The FD function at $3.5 \mathrm{~K}$ convoluted with Gaussian (broken line), fitting curves with Dynes function for s-wave (black line), and for d-wave (gray line) are shown.? 


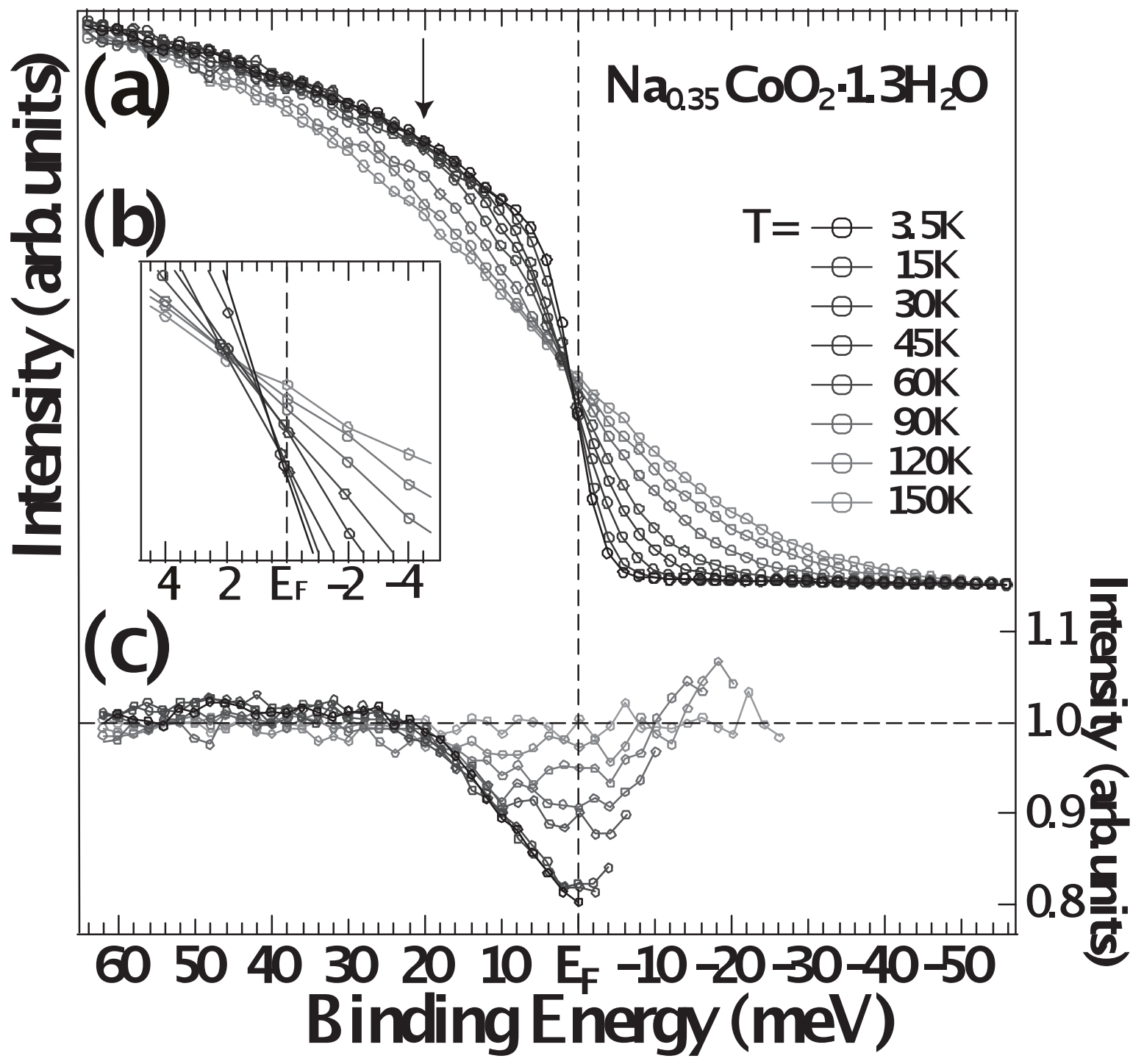

Fig.1. Shimojima et al., 


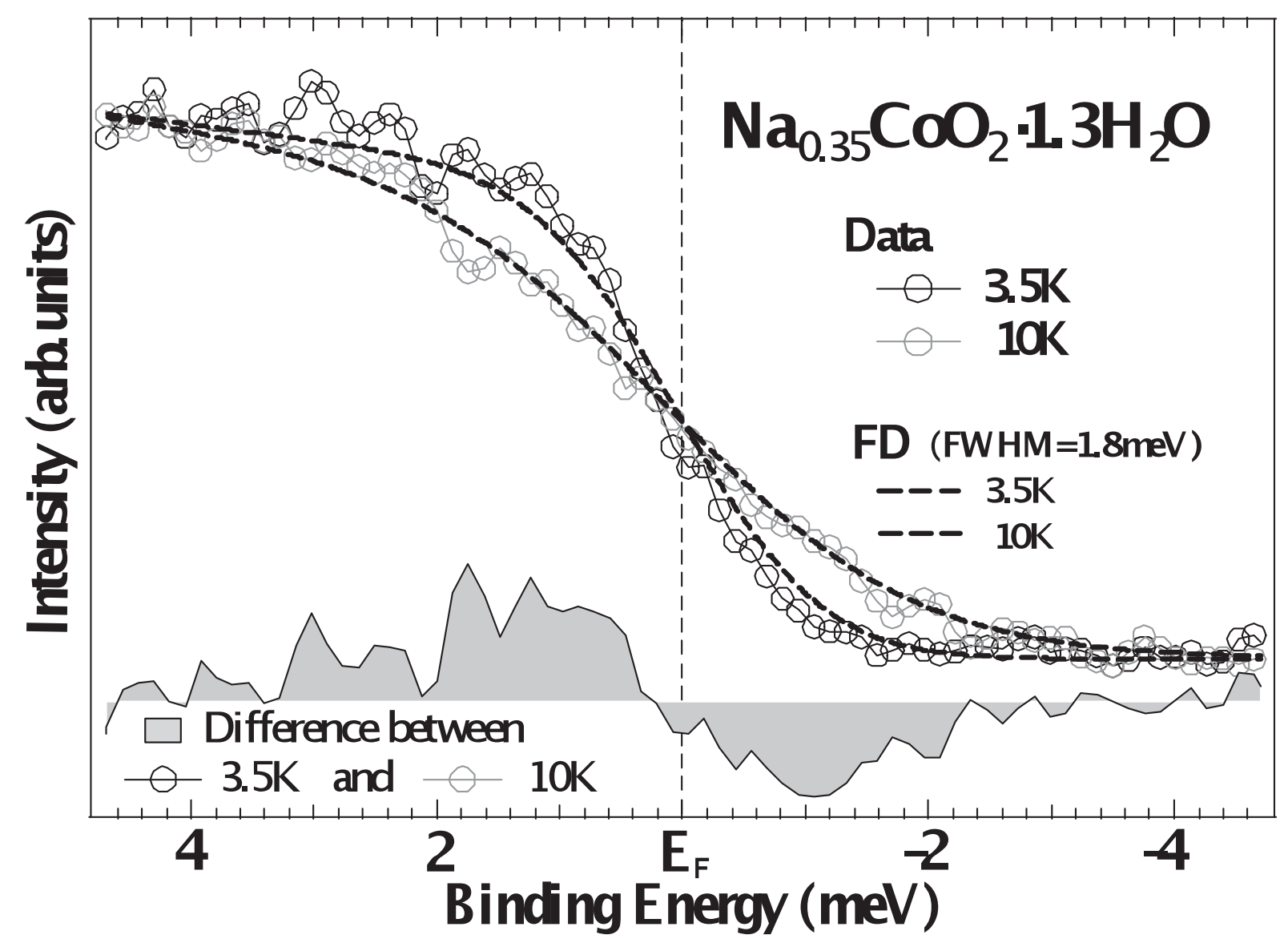

Fig.2. Shimojima et al., 


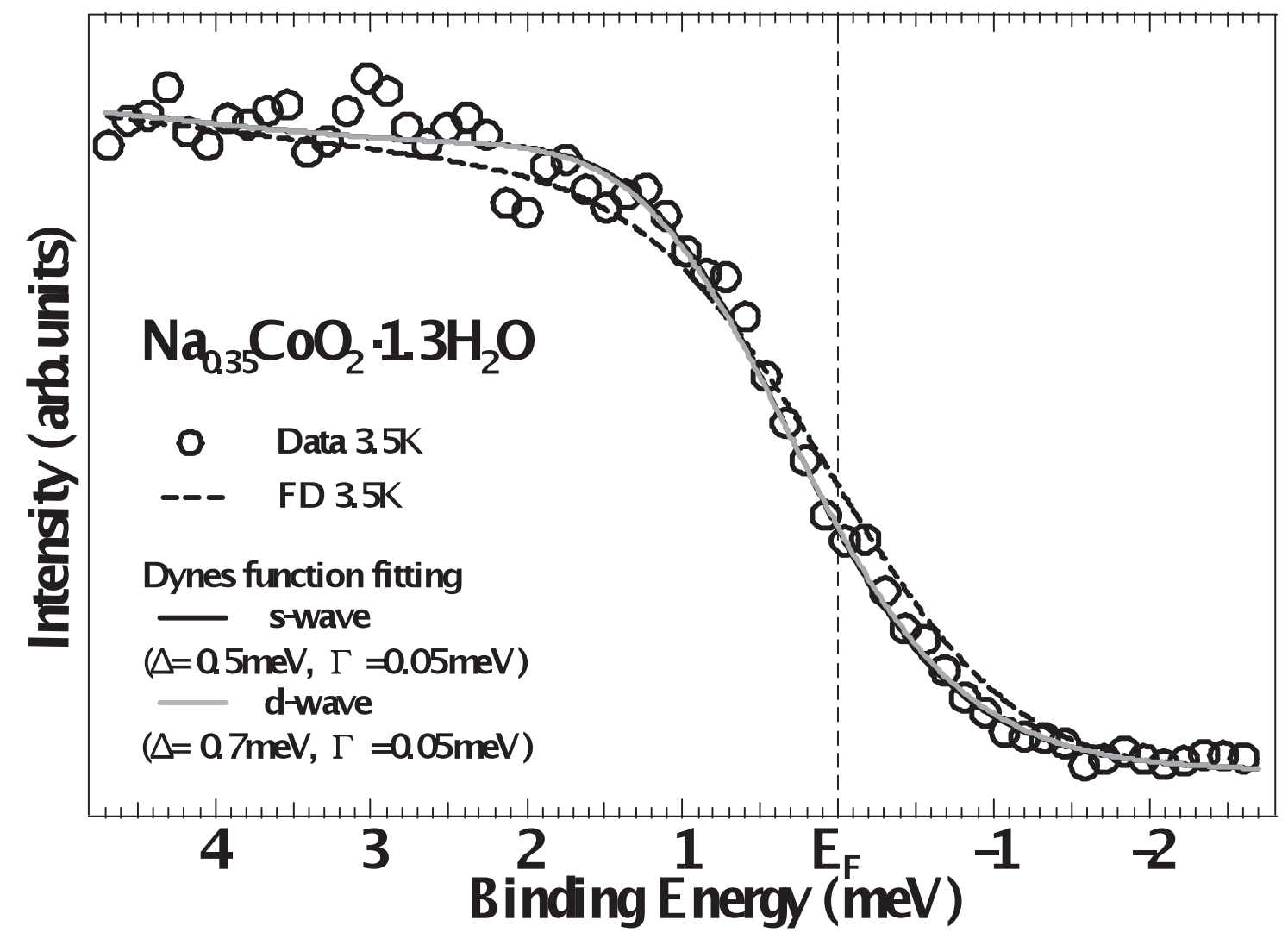

Fig.3. Shimojima et al., 